Research Article

\title{
Green's Function and Positive Solutions of a Third-Order Equation with Periodic Boundary Conditions
}

\author{
Nickolai Kosmatov \\ Department of Mathematics and Statistics, University of Arkansas at Little Rock, Little Rock, AR 72204-1099, USA \\ Correspondence should be addressed to Nickolai Kosmatov; nxkosmatov@ualr.edu
}

Received 28 January 2020; Accepted 26 February 2020; Published 1 April 2020

Academic Editor: Rodica Luca

Copyright ( 2020 Nickolai Kosmatov. This is an open access article distributed under the Creative Commons Attribution License, which permits unrestricted use, distribution, and reproduction in any medium, provided the original work is properly cited.

We apply the fixed point index to obtain positive solutions of a nonresonant periodic boundary value problem for a third-order differential equation $u^{\prime \prime \prime}+\rho^{3} u=\lambda f(u)$.

\section{Introduction}

Third-order differential equations arise in many areas of physics and engineering [1] and describe, for example, deflection of a curved beam having a constant or varying cross section, a three-layered beam, and electromagnetic waves. Boundary value problems for third-order differential equations have been studied by many authors, for example, [2-9] just to name a few. In this paper, we consider a wellknown $[10,11]$ boundary value problem:

$$
\begin{gathered}
u^{\prime \prime \prime}(t)+\rho^{3} u(t)=\lambda f(u(t)), \quad t \in(0,2 \pi), \\
u^{(i)}(0)=u^{(i)}(2 \pi), \quad i=0,1,2 .
\end{gathered}
$$

We improve the results of $[10,11]$ and obtain positive solutions using the fixed point index. The solutions to (1) and (2) will be sought in the Banach space $\mathscr{B}=C[0,2 \pi]$ endowed with the max-norm. In order to obtain positive solutions, we apply the fixed point theorem of Guo and Lakshmikantham [12] stated in Section 2. is

Green's function of $u^{\prime}(t)+\rho u(t)=0$ with $u(0)=u(2 \pi)$

$$
G_{1}(t, s)=\frac{1}{e^{2 \pi \rho}-1} \begin{cases}e^{\rho(2 \pi+s-t)}, & 0 \leq s \leq t \leq 2 \pi, \\ e^{\rho(s-t)}, & 0 \leq t \leq s \leq 2 \pi .\end{cases}
$$

Green's function of $u^{\prime \prime}(t)-\rho u^{\prime}(t)+\rho^{2} u(t)=0$ with $u^{(i)}(0)=u^{(i)}(2 \pi), i=0,1$, is

$$
\begin{aligned}
G_{2}(t, s)= & \frac{2}{\sqrt{3} \rho\left(e^{\pi \rho}+e^{-\pi \rho}-2 \cos \sqrt{3} \pi \rho\right)} \\
& \cdot \begin{cases}g_{1}(t-s), & 0 \leq s \leq t \leq 2 \pi, \\
g_{2}(s-t), & 0 \leq t \leq s \leq 2 \pi,\end{cases}
\end{aligned}
$$

where

$$
\begin{aligned}
g_{1}(x)= & e^{(1 / 2) \rho x}\left(\left(e^{-\pi \rho}-\cos \sqrt{3} \pi \rho\right) \sin \frac{\sqrt{3}}{2} \rho x\right. \\
& \left.+\sin \sqrt{3} \pi \rho \cos \frac{\sqrt{3}}{2} \rho x\right), \\
g_{2}(x)= & e^{-(1 / 2) \rho x}\left(\left(e^{\pi \rho}-\cos \sqrt{3} \pi \rho\right) \sin \frac{\sqrt{3}}{2} \rho x\right. \\
& \left.+\sin \sqrt{3} \pi \rho \cos \frac{\sqrt{3}}{2} \rho x\right),
\end{aligned}
$$

where $x \in[0,2 \pi]$. To ensure that $G_{2}(t, s) \geq 0$, we need $\rho \in(0,1 / \sqrt{3})$.

We maximize $g_{1}$ and $g_{2}$. Introduce, for convenience,

$$
\begin{aligned}
& A(\rho)=e^{-\pi \rho}-\cos \sqrt{3} \pi \rho, \\
& B(\rho)=\sin \sqrt{3} \pi \rho, \\
& C(\rho)=e^{\pi \rho}-\cos \sqrt{3} \pi \rho .
\end{aligned}
$$

We formally find that $g_{1}^{\prime}\left(x_{1}\right)=0$ if 


$$
x_{1}(\rho)=\frac{2}{\sqrt{3} \rho} \cot ^{-1} \frac{\sqrt{3} B(\rho)-A(\rho)}{\sqrt{3} A(\rho)+B(\rho)},
$$

provided $x_{1} \in(0,2 \pi)$.

Note that

$$
\begin{aligned}
\lim _{\rho \longrightarrow 0^{+}} \frac{A(\rho)}{B(\rho)} & =-\frac{1}{\sqrt{3}}, \\
\lim _{\rho \longrightarrow(1 / \sqrt{3})^{-}} \frac{A(\rho)}{B(\rho)} & =\infty .
\end{aligned}
$$

Also

$$
\left(\frac{A}{B}\right)^{\prime}(\rho)=\pi \frac{\sqrt{3}-e^{-\pi \rho}(\sin \sqrt{3} \pi \rho+\sqrt{3} \cos \sqrt{3} \pi \rho)}{\sin ^{2} \sqrt{3} \pi \rho} .
$$

\section{Denoting}

$$
\alpha(\rho)=\sqrt{3}-e^{-\pi \rho}(\sin \sqrt{3} \pi \rho+\sqrt{3} \cos \sqrt{3} \pi \rho),
$$

we have $\alpha^{\prime}(\rho)=4 \pi e^{-\pi \rho} \sin \sqrt{3} \pi \rho>0, \rho \in(0,1 / \sqrt{3})$, and $\alpha(0)=0$ and we have $(A / B)^{\prime}(\rho)>0$. Hence,

$$
\phi(\rho)=\frac{\sqrt{3} B(\rho)-A(\rho)}{\sqrt{3} A(\rho)+B(\rho)},
$$

is a decreasing function on $(0,1 / \sqrt{3}), \lim _{\rho \longrightarrow 0^{+}} \phi(\rho)=\infty$, and $\left.\lim _{\rho \longrightarrow(1 / \sqrt{3}}\right)^{-} \phi(\rho)=-1 / \sqrt{3}$. We have

$$
\begin{aligned}
\lim _{\rho \longrightarrow(1 / \sqrt{3})^{-}} x_{1}(\rho) & =\lim _{\rho \longrightarrow(1 / \sqrt{3})^{-}} \frac{2}{\sqrt{3} \rho} \cot ^{-1} \frac{\sqrt{3} B(\rho)-A(\rho)}{\sqrt{3} A(\rho)+B(\rho)}=\frac{4 \pi}{3}, \\
\lim _{\rho \longrightarrow 0^{+}} x_{1}(\rho) & =\lim _{\rho \longrightarrow 0^{+}} \frac{2}{\sqrt{3} \rho} \cot ^{-1} \phi(\rho) \\
& =-\frac{2}{\sqrt{3}} \lim _{\rho \longrightarrow 0^{+}} \frac{\phi^{\prime}(\rho)}{1+\phi^{2}(\rho)} \\
& =\frac{2}{\sqrt{3}} \lim _{\rho \longrightarrow 0^{+}} \frac{A^{\prime}(\rho) B(\rho)-B^{\prime}(\rho) A(\rho)}{A^{2}(\rho)+B^{2}(\rho)} \\
& =\frac{2}{\sqrt{3}} \lim _{\rho \longrightarrow 0^{+}} \frac{\sqrt{3}-e^{-\pi \rho}(\sin \sqrt{3} \pi \rho+\sqrt{3} \cos \sqrt{3} \pi \rho)}{e^{-2 \pi \rho}+1-2 e^{-\pi \rho} \cos \sqrt{3} \pi \rho} \\
& =\frac{2 \pi}{\sqrt{3}} \lim _{\rho \longrightarrow 0^{+}} \frac{2 \sin \sqrt{3} \pi \rho}{\cos \sqrt{3} \pi \rho+\sqrt{3} \sin \sqrt{3} \pi \rho-e^{-\pi \rho}} \\
& =\frac{2 \pi}{\sqrt{3}} \lim _{\rho \longrightarrow 0^{+}} \frac{2 \sqrt{3} \cos \sqrt{3} \pi \rho}{3 \cos \sqrt{3} \pi \rho-\sqrt{3} \sin \sqrt{3} \pi \rho+e^{-\pi \rho}} \\
& =\pi .
\end{aligned}
$$

It is a lengthy exercise to verify that $x_{1}(\rho)$ is increasing on $(0,1 / \sqrt{3})$. We infer

$$
\pi<x_{1}(\rho)<\frac{4 \pi}{3} .
$$

It follows from (8) that

$$
\begin{aligned}
& \cos \frac{\sqrt{3}}{2} \rho x_{1}=\frac{\sqrt{3} B-A}{2 \sqrt{A^{2}+B^{2}}} \\
& \sin \frac{\sqrt{3}}{2} \rho x_{1}=\frac{\sqrt{3} A+B}{2 \sqrt{A^{2}+B^{2}}} .
\end{aligned}
$$

In fact, $g_{1}^{\prime \prime}\left(x_{1}\right)<0$; thus,

$$
\begin{aligned}
g_{1}(x) \leq g_{1}\left(x_{1}\right) & =e^{(1 / 2) \rho x_{1}}\left(A \sin \frac{\sqrt{3}}{2} \rho x_{1}+B \cos \frac{\sqrt{3}}{2} \rho x_{1}\right) \\
& =\frac{\sqrt{3}}{2} e^{(1 / 2) \rho x_{1}} \sqrt{A^{2}+B^{2}} .
\end{aligned}
$$

By a similar argument, one can show that $g_{2}(x) \leq g_{2}\left(x_{2}\right)$, where

$$
x_{2}(\rho)=\frac{2}{\sqrt{3} \rho} \tan ^{-1} \frac{\sqrt{3} C(\rho)-B(\rho)}{\sqrt{3} B(\rho)+C(\rho)} \in\left(\frac{2 \pi}{3, \pi}\right),
$$

so that

$$
\begin{aligned}
g_{2}(x) \leq g_{2}\left(x_{2}\right) & =e^{-(1 / 2) \rho x_{2}}\left(C \sin \frac{\sqrt{3}}{2} \rho x_{2}+B \cos \frac{\sqrt{3}}{2} \rho x_{2}\right) \\
& =\frac{\sqrt{3}}{2} e^{-(1 / 2) \rho x_{2}} \sqrt{C^{2}+B^{2}} .
\end{aligned}
$$

We are in position to state and prove our first lemma.

Lemma 1. Green's functions $G_{1}(t, s)$ and $G_{2}(t, s)$ satisfy

$$
\begin{aligned}
M_{1}= & \max _{t, s \in[0,2 \pi]} G_{1}(t, s)=\frac{e^{2 \pi \rho}}{e^{2 \pi \rho}-1}, \\
m_{1}= & \max _{t, s \in[0,2 \pi]} G_{1}(t, s)=\frac{1}{e^{2 \pi \rho}-1}, \\
M_{2}= & \max _{t, s \in[0,2 \pi]} G_{2}(t, s)=\frac{1}{\sqrt{e^{\pi \rho}+e^{-\pi \rho}-2 \cos \sqrt{3} \pi \rho}} \\
& \cdot \max _{\{}\left\{e^{(1 / 2) \rho\left(x_{1}-\pi\right)}, e^{-(1 / 2) \rho\left(\pi-x_{2}\right)}\right\}, \\
m_{2}= & \max _{t, s \in[0,2 \pi]} G_{2}(t, s)=\frac{2 \sin \sqrt{3} \pi \rho}{\sqrt{3} \rho\left(e^{\pi \rho}+e^{-\pi \rho}-2 \cos \sqrt{3} \pi \rho\right)} .
\end{aligned}
$$

Proof. Identities (19) and (20) are easy to check.

The following identities are useful:

$$
\begin{aligned}
A^{2}+B^{2} & =e^{-2 \pi \rho}-2 e^{-\pi \rho} \cos \sqrt{3} \pi \rho+1, \\
C^{2}+B^{2} & =e^{2 \pi \rho}-2 e^{\pi \rho} \cos \sqrt{3} \pi \rho+1, \\
A^{2}+B^{2} & =e^{-2 \pi \rho}\left(C^{2}+B^{2}\right), \\
e^{\pi \rho}+e^{-\pi \rho}-2 \cos \sqrt{3} \pi \rho & =e^{-\pi \rho}\left(C^{2}+B^{2}\right) .
\end{aligned}
$$


Then, using (16) and (18), we have

$$
\begin{aligned}
& \max _{t, s \in[0,2 \pi]} G_{2}(t, s)=\frac{2}{\sqrt{3} \rho\left(e^{\pi \rho}+e^{-\pi \rho}-2 \cos \sqrt{3} \pi \rho\right)} \\
& \cdot \max \left\{g_{1}\left(x_{1}\right), g_{2}\left(x_{2}\right)\right\} \\
& =\frac{2 e^{\rho \pi \rho}}{\sqrt{3}\left(C^{2}+B^{2}\right)} \max \left\{g_{1}\left(x_{1}\right), g_{2}\left(x_{2}\right)\right\} \\
& =\frac{e^{\pi \rho}}{\rho\left(C^{2}+B^{2}\right)} \max \left\{e^{(1 / 2) \rho x_{1}} \sqrt{A^{2}+B^{2}},\right. \\
& \left.e^{-(1 / 2) \rho x_{2}} \sqrt{C^{2}+B^{2}}\right\} \\
& =\frac{e^{\pi \rho}}{\rho \sqrt{C^{2}+B^{2}}} \max \left\{e^{(1 / 2) \rho x_{1}-\rho \pi}, e^{-(1 / 2) \rho x_{2}}\right\} \\
& =\frac{e^{(1 / 2) \pi \rho}}{\rho \sqrt{e^{\pi \rho}+e^{-\pi \rho}-2 \cos \sqrt{3} \pi \rho}} \\
& \cdot \max \left\{e^{(1 / 2) \rho x_{1}-\rho \pi}, e^{-(1 / 2) \rho x_{2}}\right\} \\
& =\frac{1}{\rho \sqrt{e^{\pi \rho}+e^{-\pi \rho}-2 \cos \sqrt{3} \pi \rho}} \\
& \cdot \max \left\{e^{(1 / 2) \rho\left(x_{1}-\pi\right)}, e^{(1 / 2) \rho\left(\pi-x_{2}\right)}\right\},
\end{aligned}
$$

where $x_{1}$ and $x_{2}$ are given by (8) and (17), respectively. In addition,

$$
\begin{aligned}
\min _{x \in[0,2 \pi]} g_{1}(x) & =\min \left\{g_{1}(0), g_{1}(2 \pi)\right\}=\sin \sqrt{3} \pi \rho \\
& =\min \left\{g_{2}(0), g_{2}(2 \pi)\right\}=\min _{x \in[0,2 \pi]} g_{2}(x) .
\end{aligned}
$$

Hence,

$$
\min _{t, s \in[0,2 \pi]} G_{2}(t, s)=\frac{2 \sin \sqrt{3} \pi \rho}{\sqrt{3} \rho\left(e^{\pi \rho}+e^{-\pi \rho}-2 \cos \sqrt{3} \pi \rho\right)} .
$$

Observe that (21) and (22) are exact and improve the corresponding estimates stated in Lemma 3 of [10], namely,

$$
\frac{2 \sin \sqrt{3} \pi \rho}{\sqrt{3} \rho\left(e^{\pi \rho}+1\right)^{2}} \leq G_{2}(t, s) \leq \frac{2}{\sqrt{3} \rho \sin \sqrt{3} \pi \rho} .
$$

Indeed, $\quad\left(e^{\pi \rho}+1\right)^{2}>e^{\pi \rho}+e^{-\pi \rho}-2 \cos \sqrt{3} \pi \rho$, so (22) improves the first inequality. From (14) and (17), it is clear that

$$
\begin{aligned}
& \max \left\{e^{(1 / 2) \rho\left(x_{1}-\pi\right)}, e^{(1 / 2) \rho\left(\pi-x_{2}\right)}\right\}<e^{(1 / 2) \pi \rho}, \\
& \frac{\max \left\{e^{(1 / 2) \rho\left(x_{1}-\pi\right)}, e^{(1 / 2) \rho\left(\pi-x_{2}\right)}\right\}}{\sqrt{e^{\pi \rho}+e^{-\pi \rho}-2 \cos \sqrt{3} \pi \rho}} \\
& <\frac{1}{\sqrt{1+e^{-2 \pi \rho}-2 e^{-\pi \rho} \cos \sqrt{3} \pi \rho}} \\
& =\frac{1}{\sqrt{\left(e^{-\pi \rho}-\cos \sqrt{3} \pi \rho\right)^{2}+\sin ^{2} \sqrt{3} \pi \rho}}<\frac{2}{\sqrt{3} \sin \sqrt{3} \pi \rho},
\end{aligned}
$$

that is, (21) is preferred to the constant in the second inequality.

Now, Green's function of $u^{\prime \prime \prime}(t)+\rho^{3} u(t)=0$, $u^{(i)}(0)=u^{(i)}(2 \pi), i=0,1,2$, is determined from (3)-(6) by $G(t, s)=\int_{0}^{2 \pi} G_{1}(t, \tau) G_{2}(\tau, s) \mathrm{d} \tau=L_{1} H_{1}(t, s)+L_{2} H_{2}(t, s)$,

where

$$
\begin{aligned}
H_{1}(t, s) & = \begin{cases}e^{(1 / 2) \rho(t-s)}\left(\sin \left(\frac{\sqrt{3}}{2} \rho(t-s)-\frac{\pi}{6}\right)-e^{\pi \rho} \sin \left(\frac{\sqrt{3}}{2} \rho(t-s-2 \pi)-\frac{\pi}{6}\right)\right), & s \leq t, \\
e^{(1 / 2) \rho(t-s+2 \pi)}\left(\sin \left(\frac{\sqrt{3}}{2} \rho(t-s+2 \pi)-\frac{\pi}{6}\right)-e^{\pi \rho} \sin \left(\frac{\sqrt{3}}{2} \rho(t-s)-\frac{\pi}{6}\right)\right), & t \leq s,\end{cases} \\
H_{2}(t, s) & = \begin{cases}e^{\rho(s-t)}, & s \leq t, \\
e^{\rho(s-t-2 \pi)}, & t \leq s,\end{cases} \\
L_{1} & =\frac{2}{3 \rho^{2}\left(1+e^{2 \pi \rho}-2 e^{\pi \rho} \cos \sqrt{3} \pi \rho\right)},
\end{aligned}
$$


One can find (29) in [11]. The structure of (29) is too complex to be analyzed the same way as $G_{2}(t, s)$. Instead, by Lemma 1,

$$
G(t, s)=\int_{0}^{2 \pi} G_{1}(t, \tau) G_{2}(\tau, s) \mathrm{d} \tau \leq M_{2} \int_{0}^{2 \pi} G_{1}(t, \tau) \mathrm{d} \tau=\frac{M_{2}}{\rho},
$$

and, similarly,

$$
G(t, s) \geq \frac{m_{2}}{\rho} .
$$

Also,

$$
\begin{aligned}
G(t, s) & =\int_{0}^{2 \pi} G_{1}(t, \tau) G_{2}(\tau, s) \mathrm{d} \tau \leq M_{1} \int_{0}^{2 \pi} G_{2}(t, \tau) \mathrm{d} \tau=\frac{M_{1}}{\rho^{2}} \\
G(t, s) & \geq \frac{m_{1}}{\rho^{2}} .
\end{aligned}
$$

Thus,

$$
\frac{1}{\rho^{2}} \max \left\{m_{1}, \rho m_{2}\right\}=m \leq G(t, s) \leq M=\frac{1}{\rho^{2}} \min \left\{M_{1}, \rho M_{2}\right\} \text {. }
$$

Comparing $m_{1}$ to $\rho m_{2}$ and $M_{1}$ to $\rho M_{2}$, respectively, we find that the constants "compete" and we cannot simplify (34). Finally, note that Theorem 2.6 in [11] contains similar estimates only for $0<\rho<(2 / 3 \sqrt{3})$ while (34) is fulfilled for $0<\rho<(1 / \sqrt{3})$.

In the next section, as an application of (34), we obtain a positive solution of (1) and (2).

\section{Main Results}

Assume that $\left(H_{1}\right) f:[0, \infty) \longrightarrow[0, \infty)$ is continuous and $\lambda>0$.

It is clear that the map $T: \mathscr{B} \longrightarrow \mathscr{B}$ defined by

$$
T u(t)=\lambda \int_{0}^{2 \pi} G(t, s) f(u(s)) \mathrm{d} s
$$

is completely continuous. Define

$$
\mathscr{C}=\left\{u \in \mathscr{B}: u(t) \geq \frac{m}{M}\|u\|\right\} .
$$

Obviously, $\mathscr{C}$ is a cone in $\mathscr{B}$. By (34), T: $\mathscr{C} \longrightarrow \mathscr{C}$ and $u \in \mathscr{B}$ is a solution of (1) and (2) if $u \in \mathscr{B}$ is a fixed point of $T$.

Let

$$
\begin{aligned}
\mathscr{C}_{R} & =\{u \in \mathscr{C}:\|u\|<R\}, \\
\partial \mathscr{C}_{R} & =\{u \in \mathscr{C}:\|u\|=R\} .
\end{aligned}
$$

We apply the following theorem [12].

Theorem 1. Let $\mathscr{B}$ be a Banach space. Assume that $T: \overline{\mathscr{C}}_{r} \longrightarrow \mathscr{C}$ is completely continuous such that $T u \neq u$ for $u \in \partial \mathscr{C}_{r}$.

$\left(A_{1}\right)$ If $\|T u\| \geq\|u\|$ for $u \in \partial \mathscr{C}_{r}$, then $i\left(T, \mathscr{C}_{r}, \mathscr{C}\right)=0$.

$$
\left(A_{2}\right) \text { If }\|T u\| \leq\|u\| \text { for } u \in \partial \mathscr{C}_{r} \text {, then } i\left(T, \mathscr{C}_{r}, \mathscr{C}\right)=1 \text {. }
$$

We will restrict our attention to the case.

$\left(H_{2}\right) \quad f_{0}, f_{\infty} \neq 0$, where $f_{0}=\lim _{x \longrightarrow 0} f(x) / x$ and $f_{\infty}=\lim _{x \longrightarrow \infty} f(x) / x$.

Recall that

$$
\int_{0}^{2 \pi} G(t, s) \mathrm{d} s=\frac{1}{\rho^{3}}
$$

If there exists $\delta>0$ such that $f(x) \geq \delta x$, then for $u \in \mathscr{C}$,

$$
\begin{aligned}
T u(t) & =\lambda \int_{0}^{2 \pi} G(t, s) f(u(s)) \mathrm{d} s \\
& \geq \lambda \delta \int_{0}^{2 \pi} G(t, s) u(s) \mathrm{d} s \\
& \geq \lambda \delta \frac{m}{M} \int_{0}^{2 \pi} G(t, s) \mathrm{d} s\|u\| \\
& =\lambda \frac{\delta m}{M \rho^{3}}\|u\| .
\end{aligned}
$$

Similarly, if there exists $\delta>0$ such that $f(x) \leq \gamma x$, then, for $u \in \mathscr{C}$,

$$
\begin{aligned}
T u(t) & =\lambda \int_{0}^{2 \pi} G(t, s) f(u(s)) \mathrm{d} s \\
& \leq \lambda \gamma \int_{0}^{2 \pi} G(t, s) u(s) \mathrm{d} s \\
& \leq \lambda \gamma \int_{0}^{2 \pi} G(t, s) \mathrm{d} s\|u\| \\
& =\lambda \frac{\gamma}{\rho^{3}}\|u\| .
\end{aligned}
$$

From these inequalities, by Theorem 1, we have the following result.

Theorem 2. If $\left(H_{1}\right)$ and $\left(H_{2}\right)$ are satisfied, then (1) and (2) have at least one positive solution provided:

$$
\lambda \in\left(\frac{M \rho^{3}}{m \max \left\{f_{\infty}, f_{0}\right\}}, \frac{\rho^{3}}{\min \left\{f_{\infty}, f_{0}\right\}}\right) .
$$

Proof. Consider $f_{\infty}>f_{0}$. Let $f_{\infty}>\epsilon>0$ be such that

$$
\lambda \in\left(\frac{M \rho^{3}}{m\left(f_{\infty}-\epsilon\right)}, \frac{\rho^{3}}{f_{0}+\epsilon}\right) .
$$

There exists $R_{1}<0$ such that $f(x) \leq\left(f_{0}+\epsilon\right) x$, $x \in\left[0, R_{1}\right]$, that is, $f(u(t)) \leq\left(f_{0}+\epsilon\right) u(t), u \in \partial \mathscr{C}_{R_{1}}$. Hence,

$$
\|T u\| \leq \lambda\left(f_{0}+\epsilon\right) \frac{1}{\rho^{3}}\|u\|<\|u\|, \quad u \in \partial \mathscr{C}_{R_{1}} .
$$

There exists $R>R_{1}$ such that $f(x) \geq\left(f_{\infty}-\epsilon\right) x, x \geq R$. Let $R_{2}=\max \left\{2 R_{1}, M R / m\right\}$. Then, $u(t) \geq(m / M)\|u\| \geq R$, $u \in \partial \mathscr{C}_{R_{2}}$. As above, for $u \in \partial \mathscr{C}_{R_{2}}$, 


$$
\|T u\| \geq \lambda\left(f_{\infty}-\epsilon\right) \frac{m}{M \rho^{3}}\|u\|>\|u\| .
$$

It follows that $i\left(T, \mathscr{C}_{R_{1}}, \mathscr{C}\right)=1$ and $i\left(T, \mathscr{C}_{R_{2}}, \mathscr{C}\right)=0$ so that $i\left(T, \mathscr{C}_{R_{2}} / \overline{\mathscr{C}}_{R_{1}}, \mathscr{C}\right)=-1$, that is, $T$ has a fixed point in $\mathscr{C}_{R_{2}} / \overline{\mathscr{C}}_{R_{1}}$.

The case $f_{\infty}<f_{0}$ is handled similarly.

\section{Data Availability}

No data were used to support this study.

\section{Conflicts of Interest}

The authors declare that they have no conflicts of interest.

\section{References}

[1] M. Gregus, Third Order Linear Differential Equations, Series: Mathematics and its Applications, Vol. 22, Springer, Dordrecht, Netherlands, 1987.

[2] Y. Chen and J. Yan, "Positive solutions for third-order Sturm-Liouville boundary value problems with $p$-Laplacian," Computers \& Mathematics with Applications, vol. 59, pp. 2059-2066, 2010.

[3] A. Dang Quang, L. Dang Quang, and N. T. K. Quy, "A novel efficient method for nonlinear boundary value problems," Numerical Algorithms, vol. 76, no. 2, pp. 427-439, 2017.

[4] J. R. Graef and B. Yang, "Positive solutions of a third order nonlocal boundary value problem," Discrete \& Continuous Dynamical Systems-S, vol. 1, no. 1, pp. 89-97, 2008.

[5] R. Hakl, "Periodic boundary-value problem for third-order linear functional differential equations," Ukrainian Mathematical Journal, vol. 60, no. 3, pp. 481-494, 2008.

[6] Y. Li and Q. Li, "Positive periodic solutions of third-order ordinary differential equations with delays," Abstract and Applied Analysis, vol. 2014, Article ID 547683, 8 pages, 2014.

[7] D. Luo, "Existence of positive solutions of a third order nonlinear differential equation with positive and negative terms," Advances in Difference Equations, vol. 2018, p. 87, 2018.

[8] Z. Y. Wang and Y. C. Mo, "Bifurcation from infinity and multiple solutions of third order periodic boundary value problems," Applied Mathematics E-Notes, vol. 12, pp. 118-128, 2012.

[9] Y. Sun, "Positive solutions of singular third-order three-point boundary value problem," Journal of Mathematical Analysis and Applications, vol. 306, no. 2, pp. 589-603, 2005.

[10] L. Kong, S. Wang, and J. Wang, "Positive solution of a singular nonlinear third-order periodic boundary value problem," Journal of Computational and Applied Mathematics, vol. 132, no. 2, pp. 247-253, 2001.

[11] J. Ren, S. Siegmund, and Y. Chen, "Positive periodic solutions for third-order nonlinear differential equations," Electronic Journal of Differential Equations, vol. 2011, no. 66, pp. 1-19, 2011.

[12] D. Guo and V. Lakshmikantham, Nonlinear Problems in Abstract Cones, Academic Press, Orlando, FL, USA, 1988. 